\title{
A Vehicular Electronic Image Stabilization System Based on A Gasoline Model Car Platform
}

\author{
Yuan Yang \\ Southeast University \\ Jian-Hua Wu \\ Southeast University \\ Zi-Qian Zhao \\ Southeast University \\ Guo-Dong Yin \\ Southeast University
}

Ning Zhang ( $\square$ nzhang_cn@seu.edu.cn )

Southeast University https://orcid.org/0000-0002-3397-2522

\section{Original Article}

Keywords: electronic image stabilization, environment perception system, feature point, adaptive Kalman filter, gasoline model car

Posted Date: October 12th, 2020

DOl: https://doi.org/10.21203/rs.3.rs-88810/v1

License: (c) (i) This work is licensed under a Creative Commons Attribution 4.0 International License. Read Full License 


\section{Title page}

A vehicular electronic image stabilization system based on a gasoline model car platform 


\section{ORIGINAL ARTICLE}

\section{A vehicular electronic image stabilization system based on a gasoline model car platform}

Ning Zhang ${ }^{1} \cdot$ Yuan Yang ${ }^{2} \cdot$ Jian-Hua $W^{1} \cdot$ Zi-Qian Zhao ${ }^{1} \cdot$ Guo-Dong Yin ${ }^{1}$

${ }^{1}$ Department of Vehicle Engineering, School of Mechanical Engineering, Southeast University, Nanjing, 211189, China

${ }^{2}$ School of Instrument Science and Engineering, Southeast University, Nanjing, 210096, China

(C) Chinese Mechanical Engineering Society and Springer-Verlag Berlin Heidelberg 2017

\begin{abstract}
The image sequence captured by the vehicular camera vibrates due to the vehicle vibration or harsh road conditions. It greatly affects the subsequent image processing. As a video processing technology, electronic image stabilization (EIS) can solve this problem effectively. In this paper, a gasoline model car platform is established, which has rich vibration characteristics. Besides, high unevenness roads are selected to make the vibration problem more prominent. Considering vehicle demand, feature point detection and matching algorithm is applied to match images in the process of establishing EIS. In addition, the algorithm of eliminating mismatched feature points and the adaptive Kalman filter (AKF) is improved to make them more suitable for vehicle application. The experimental results show that the EIS developed in this paper meets the real-time requirements and can work normally in harsh road conditions. Since the platform and working scenarios are more extreme than the normal conditions, the EIS developed in this paper has high portability in the environment perception system of smart car.
\end{abstract}

Keywords: electronic image stabilization, environment perception system, feature point, adaptive Kalman filter, gasoline model car,

\section{Introduction}

When the vehicle is running, various vibration conditions are inevitable, which have a certain negative impact on the subsequent image processing algorithms. This problem can be solved by installing a mechanical damping structure or by EIS. Since EIS is based on the image processing method, which costs less than adding additional mechanical structure, it has become a common way to solve this problem. The related researches can be dated back to 1980s, Jean [1] developed an EIS for the reconnaissance vehicle realizing at the resolution of $640 * 480$

EIS is a computer vision processing technology that uses image processing algorithms to weaken or even eliminate random jitter in the video. The processes are mainly divided into three steps: 1. Estimate the transformation matrix of the current frame relative to the reference frame; 2. Filter the state variables derived by transformation matrix; 3 . Inverse compensation and output of the image [2].

Estimation of the transformation matrix is the most important step. The methods mainly include block matching method, phase correlation matching method, bit plane matching method, gray projection method, feature matching method, optical flow method [3].

In the feature matching method, feature information includes point features, line features, edge features, etc. The point features is applied widely. The Harris feature point detection algorithm was first proposed by Harris [4] in 1988. It performs convolution calculation on the image through the derivative of the Gaussian function. Harris algorithm is relatively stable when dealing with rotation and changing brightness, but it does not have scale invariance. David et al. [5] proposed scale-invariant feature transform (SIFT), which has excellent scale invariance and has been widely used in related fields. Bay [6] proposed the Speeded Up Robust Features algorithm (SURF), which is based on the SIFT. This algorithm uses a fast Hessian matrix to detect feature points, and uses an integral image method to reduce the calculation time, which makes the efficiency of the algorithm great improve. Features from Accelerated Segment Test (FAST) algorithm is proposed by Edward [7] in 2006, it is based on the Susan algorithm and determine the feature point by detecting the pixel values around the image. These four feature point extraction algorithms are the most widely used methods. Various EIS algorithms are developed based on these four methods [8-11].

Filter is another important step to influence the effect of EIS. More commonly used filter algorithms in EIS include mean filter, least square fitting filter, B-spline 
curve fitting filter, Kalman filter (KF). Mean filtering and least square filtering cannot meet real-time requirements. The method based on B-spline curve relies on kinematic model [12]. The method based on KF has become the mainstream method in various research [13-15]. However, the effect of KF is sensitive to the system's noise parameter settings, the frequency and amplitude of random motion, etc. Researchers have done a lot of work on this problem. Park [16] proposes a new image stabilization method based on finite impulse response filter, which is more robust against mistuning on the model parameters than the KF. Choi [17] used Extend Kalman Filter in aerial airborne imaging to remove the jitter of the camera and retain scanning motion. Yang [18] proposed a novel stabilization algorithm based on particle filter in EIS, Zhu [19] made further improvements based on his research. Besides, Ioannidis [20] proposed the basic features of the Hilbert-Huang Transform in order to separate the local motion signal, it is a novel method in the field of EIS.

From the above research, the following research trends and shortcomings can be summarized: 1. Various types of EIS have many different methods, they are mostly used in handheld recording equipment and are rarely built from the perspective of vehicles; 2 . The filtering has a great influence on the performance of the EIS. Irregular road surface excitation is easy to cause the filtering to diverge, especially in the KF with fixed system noise. This is rarely mentioned in the existing research; 3 . The real-time nature of electronic image stabilization has become a concern of many researchers, especially in the feature detection and filtering. However, the real-time nature of the matching process has not been paid close attention to. The image sequence captured by the vehicular camera vibrates duo to the vehicle vibration or poor road conditions. This paper focuses on the research of EIS to solve this problem. Since this paper pays close attention to image sequence jitter caused by vibration, a gasoline model car platform is established in Section 2, which has rich vibration characteristics. Besides, harsh road conditions are selected to make the vibration problem more prominent. Furthermore, feature point detection algorithm is used to match two adjacent frames in Section 3 . Then, the algorithm of eliminating mismatched feature points and the $\mathrm{AKF}$ are improved respectively to make them more suitable for vehicle use. Finally, conclusions are provided in Section 6.

\section{Experimental platform}

\subsection{Structure of the model car}

The structure of model car is shown as Figure 1. Except a middle mounted engine, the chassis structure of the gasoline model car is similar to that of the ordinary gasoline car. The model car is mainly controlled by two control servos, namely the steering servo and the throttle servo. The structural layout of this model car is shown in Figure 1. The control system is placed in the front of the model car, and a wheel speed sensor, in the form of an encoder, is installed under the chassis.

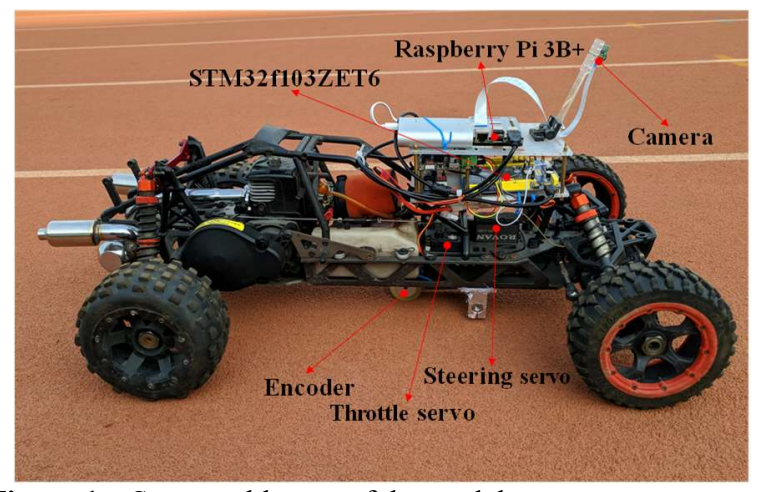

Figure 1 Structural layout of the model car.

\subsection{EE architecture of the platform}

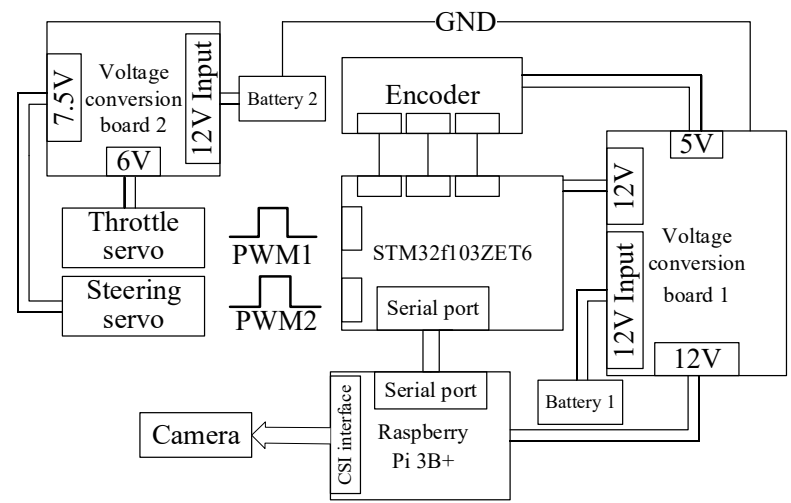

Figure 2 EE architecture of the platform. 
Figure 2 shows the EE architecture of the platform. In the computing platform, the upper computer uses Raspberry Pi $3 \mathrm{~B}+$ for capturing video. The photo sensitive chip of the camera used in this experiment is Sony IMX219, which belongs to a CMOS type chip. The camera captures video at the resolution of $480 * 480$. The STM32 microcomputer is used to control the model gasoline car through two PWM signals of the single-chip pins. Due to the load limitation of the model car. Electronic image stabilization adopts offline calculation method, the calculation platform is a quad-core CPU, and the basic frequency is $3.2 \mathrm{GHz}$.

\subsection{Experimental road conditions}

Since this platform uses an encoder to collect speed signals, it is difficult for the encoder to work stably under harsh road conditions. Therefore, during the experiment, we installed bumps on both front wheels to instead the high unevenness road, which is shown in Figure 3(a) and Figure 3(b). From the perspective of capturing video, this is equivalent to conducting experiments directly under harsh road conditions.

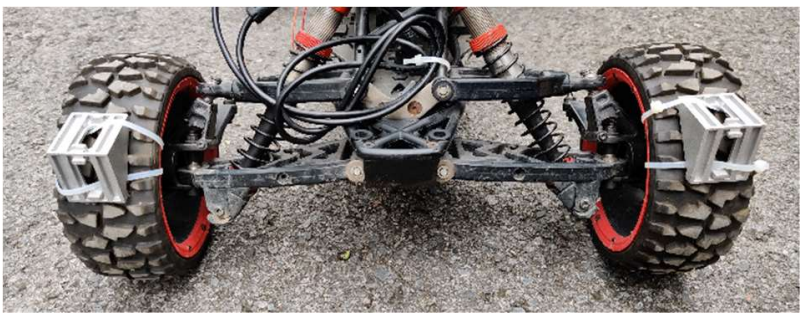

Figure 3(a) Front wheels of the model car.

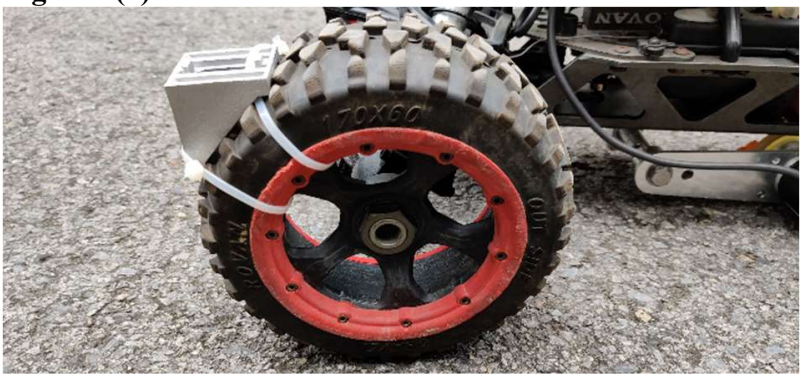

Figure 3(b) Left front wheel of the model car.

\section{Feature point detection based on ORB algorithm}

\subsection{Selection of image matching algorithm}

As mentioned in Section I, image matching is the first and one of most important step of EIS. At present, a large number of algorithms for detecting motion vectors between frames have been proposed. Commonly used are: block matching algorithm, gray projection algorithm, optical flow algorithm and feature matching algorithm. Optical flow method and gray projection method cannot adapt to complex scene changes. Block matching algorithm and feature matching method are the better choice for the vehicle demand. For block matching algorithm, to ensure the unique needs of the vehicle, a larger search area size for the matching blocks is required. Otherwise, the search results may fall into the local optimum easily. However, the search area size for the matching blocks has a positive correlation with search time. Under the real-time requirements of the vehicle, the accuracy of the block matching algorithm is limited. Based on the above discussion, feature matching algorithm is the most suitable for vehicle demands.

It has been mentioned in Section I, Harris, SIFT, SURF and ORB are the most widely used feature point extraction algorithm. The processing time of one frame at the resolution of $480 * 480$ pixels using these algorithms are shown as Table 1.

Table 1 Comparison of the processing time

\begin{tabular}{lrrrr}
\hline Algorithm & \multicolumn{1}{c}{ Harris } & \multicolumn{1}{c}{ SIFT } & \multicolumn{1}{c}{ SURF } & \multicolumn{1}{c}{ ORB } \\
\hline Number of feature points & 481.7 & 502.8 & 566.1 & 483.7 \\
Processing time $(\mathrm{ms})$ & 65.3 & 507.3 & 256.9 & 6.0 \\
\hline
\end{tabular}

From the point of processing time, ORB algorithm has significant advantage. Besides, the number of extracted points is no less than other algorithms.

\subsection{ORB algorithm principle}

ORB algorithm is the combination of FAST and improved Binary Robust Independent Elementary Features algorithm (BRIEF), which are used to detect and describe the feature [21].

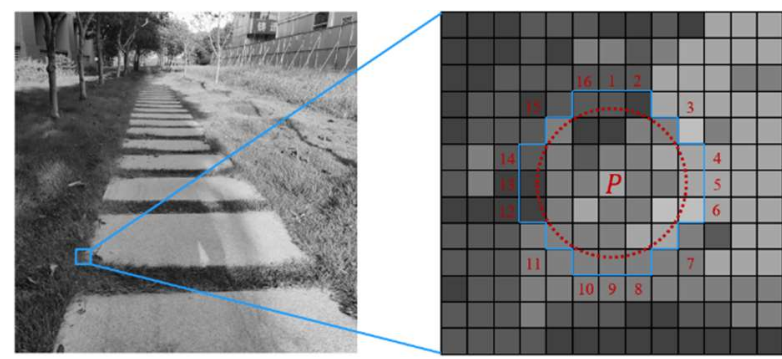

Figure 4 Corner point and the 16 points around it. 
ORB algorithm find key points in the image, such as corner points. Usually, they have the characteristic of sharply changing pixel values from light to dark. As shown in Figure 4, by comparing the gray value of point $\mathrm{P}$ with the 16 points' gray values around it, whether $\mathrm{P}$ is a corner point is determined.

Then, the corresponding feature vector of each key point is calculated. The feature vector created by the ORB algorithm contains only 1 and 0 , which is called a binary feature vector. The order of 1 and 0 depends on the specific key point and the pixel area around it. This vector represents the intensity pattern around the key point, so multiple feature vectors can be used to identify a larger area or even a specific object in the image. Define [21]:

$$
\text { descriptor }(X, Y, i)= \begin{cases}1 & I(X) \geq I(Y) \\ 0 & I(X)<I(Y)\end{cases}
$$

$X$ denotes the feature point detected, $Y$ denotes the points to be compared. I denotes the gray value of the point. $i$ represents the ith bit in the BRIEF description. Connect the bits of the $N$ pixels, a bit string is obtained. To solve the problem that the BRIEF not defining the main direction, BRIEF is improved in ORB by gray centroid method:

$$
m_{p q}=\sum_{x, y} x^{p} y^{q} I(x, y)
$$

$x, y$ are the pixel coordinates in the neighborhood around the feature point, the grayscale centroid can be determined by:

$$
\theta=\arctan \left(\frac{m_{01}}{m_{10}}\right)
$$

Therefore, ORB has rotation invariance, which is essential for the demand of on-board working condition.

\subsection{Result of feature points detection and matching}

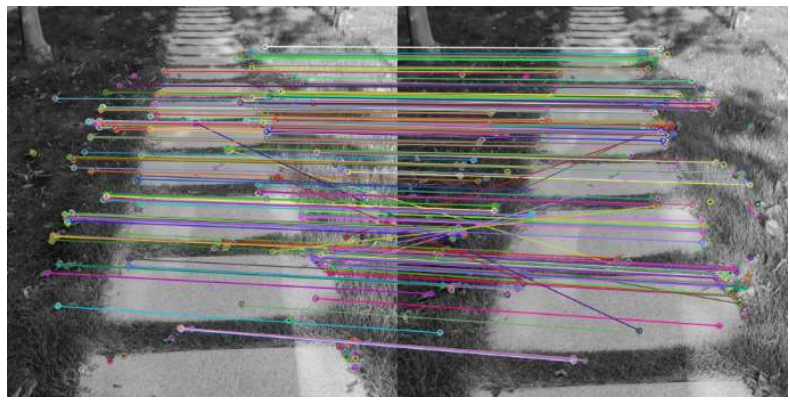

Figure 5 Feature point detection and matching results.

It is found that the average time of feature point detection is $6.01 \mathrm{~ms}$, the average detected points of each frame is 483.7. The average amount of matched feature point pairs is 223.4. It should be noted that there are some mismatched points shown in Figure 5.

\section{Elimination of mismatched feature points}

\subsection{Image transformation matrix}

The essence of judging whether a point pair is mismatched is to judge whether this point pair obeys to the image transformation matrix $H$. Therefore, it needs to be determined before the mismatched points are eliminated.

Choosing an appropriate motion model to calculate the global motion vector can ensure a better image stabilization effect. The more complex model can accurately describe the image motion, but the amount of calculation increases.

Considering the possible working conditions of vehicle body shaking with large scale. The six-parameter affine model is used to describe the rotation and translation motion well and ensure good prediction accuracy. The model is as follows:

$$
\left(\begin{array}{l}
x_{2} \\
y_{2}
\end{array}\right)=s\left(\begin{array}{rr}
\cos \Delta \theta & -\sin \Delta \theta \\
\sin \Delta \theta & \cos \Delta \theta
\end{array}\right)\left(\begin{array}{l}
x_{1} \\
y_{1}
\end{array}\right)+\left(\begin{array}{l}
\Delta x \\
\Delta y
\end{array}\right)
$$

In the equation above, $\left(x_{1}, y_{1}\right)$ and $\left(x_{2}, y_{2}\right)$ represent the coordinates of the reference frame and the current frame respectively. $\Delta \theta$ represents the roll angle between two frames. $\Delta x$ is the lateral displacement of the current frame relative to the reference frame, while $\Delta y$ denotes the vertical displacement.

\subsection{Improvement of RANSAC algorithm}

Based on the matched feature point, the transformation matrix of the current frame relative to the reference frame can be derived by fitting method like Least Squares Fitting. However, the accuracy of transformation matrix is affected by the mismatched feature points. The Random Sampling Consensus algorithm (RANSAC) divides all points into two types: inliers and outliers. Inliers refer to points which can satisfy the model, while the outliers refer to the interference point. In this way, it prevents the calculation results from being affected by outliers.

The specific implementation process of RANSAC 
algorithm is shown as follows [22]:

Step 1: Set the minimum number $s$ of point pairs which can be used to derive $H$ of two frames. Select $s$ pairs of points without repeating to form a point set $S_{r}$.

Step 2: Set the number of iterations $k$. Suppose the amount of data is $n$, the amount of inliers is $m$. Obviously, the probability that all points in set $S_{r}$ are all inliers is:

$$
w=\frac{m}{n} \cdot \frac{m-1}{n-1} \cdot \cdots \cdot \frac{m-s+1}{n-s+1}
$$

Within the number of iterations $k$, the probability that at least one $S_{r}$ only contains inliers is $p$. Then, the relationship of $k$ and $p$ is given as:

Then, $\mathrm{k}$ is derived as:

$$
(1-\mathrm{w})^{k} \leq 1-p
$$

$$
k \geq \frac{\log (1-p)}{\log (1-w)}
$$

Step 3: Determine the number of inliers that satisfy $H$, the judgment criteria is:

$$
\left\|p_{i}{ }^{\prime}-H p_{i}\right\|<e
$$

In the above equation, $p_{i}{ }^{\prime}$ and $p_{i}$ represent the coordinate of the current frame and the reference frame. $e$ refers to the error set to distinguish inliers and outliers. The total number of liners is counted as $M$.

Step 4: The transformation matrix $H$ corresponding to the maximum $M$ is the best matrix to be found.

Although RANSAC algorithm has certain robustness, few defects exist in practical engineering: 1 . If the matching accuracy is not high enough, a large number of outliers leads to an increase in the number of iterations. 2. If the random points are too concentrated, the accuracy of transformation matrix is affected seriously. 3. If the selected feature points contain outliners, the entire iteration is also be performed once, which greatly wastes calculation time.

Based on the shortcomings above, the RANSAC algorithm is improved in this paper. The specific implementation steps are:

Step 1: Rank the point pairs by Hamming distance $D_{H}$. Remove this pair of points, if:

$$
D_{H}>E+K * \sigma \text { or } D_{H}<E-K * \sigma
$$

In the equation above, $E$ denotes the mean Hamming distance of points while $\sigma$ denotes their variance. $K$ can be used to adjust the amount of removed points.
Step 2: Set the minimum number $s$ of point pairs which can be used to derive $H$. Select $s$ pairs of points without repeating in different grids to form a set $S_{r}$.

Step 3: Calculate the number of iterations.

Step 4: Select 3 pairs of points. Determine the amount of inliers. If the amount is less than 2 , jump out of this iteration and proceed to the next iteration.

Step 5: The transformation matrix $H$ corresponding to the maximum $M$ is the best matrix to be found.

\subsection{Result of eliminating mismatched feature points}

As we all know, when the car is stopped, the transformation matrix between two frames should be identity matrix. However, the running cars, walking people or even shaking leaves exert some mismatch points, which changes the transformation matrix from identity matrix. Using this feature, we can detect the performance of improved RANSAC performance.

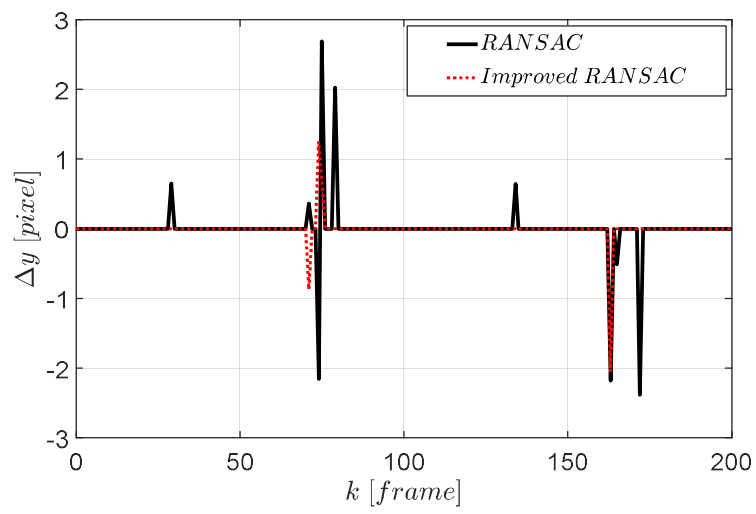

Figure 6 Matching results of the improved RANSAC.

Define the matching accuracy $A_{m}$ as:

$$
A_{m}=\frac{\left|\Delta y_{k}\right|}{k_{\max }} \quad k \in[1,200]
$$

$k_{\text {max }}$ denotes the total number of frames.

Obviously, $\Delta y$ should be 0 theoretically. It indicates that the $A_{m}$ closer to 0 corresponds to a better algorithm. Since the $A_{m}$ of RANSAC and the improved RANSAC is 0.068 and 0.020 respectively, the improved RANSAC proposed in this paper has better performance. 


\section{Filter of the transformation matrix}

\subsection{Kalman Filter}

Commonly used filter in EIS include mean filtering, least square fitting filtering, B-spline curve fitting filtering and KF. Mean filtering and least-squares filtering require multiple frames of image observation values. Therefore, their implementation has lag, which makes it difficult to meet vehicle requirements. The method based on the B-spline curve relies on the kinematics model. The method based on KF has become the mainstream method in this research field.

In the classic $\mathrm{KF}$, both system noise $Q$ and observation noise $R$ need to be set in advance. As shown in Figure 7, the filter effect is completely different when the two sets of data are $Q=0.01, R=0.1$ and $Q=0.1, R=0.1$ respectively. Therefore, considering vehicle conditions, the fixed noise matrix does not have the ability to adapt to various vibrations, especially concerning extreme dynamics.

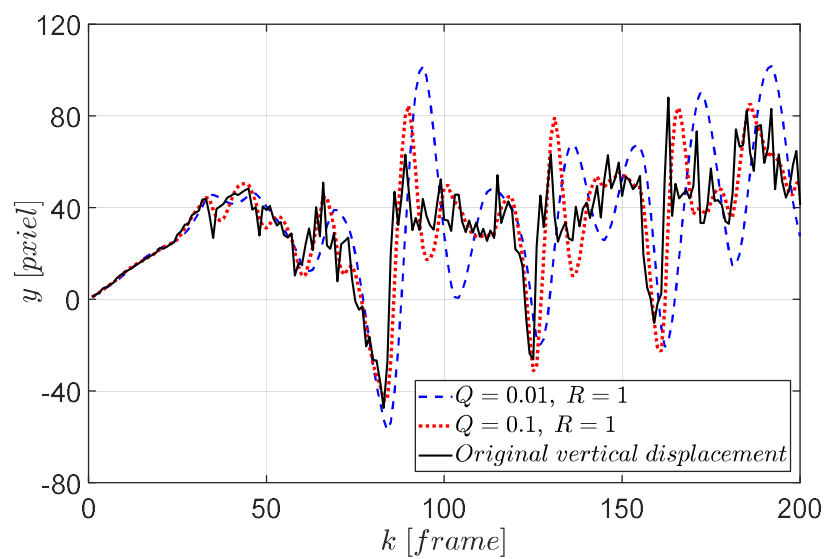

Figure 7 The effect of KF under different $\mathrm{Q}$ and $\mathrm{R}$.

\subsection{Adaptive Kalman Filter}

In order to solve this problem, the Adaptive Kalman Filter (AKF) is used to improve the adaptability of vehicular EIS. AKF corrects the parameters of the model and the noise covariance matrix. In this way, AKF reduces the influence of model error when predicting the state variables. This article mainly introduces the Sage-Husa AKF algorithm [23] into the research of EIS and makes certain improvements to it.

In $\mathrm{KF}$, the state equation and observation equation are given as:

$$
\begin{gathered}
X_{k}=\emptyset_{k, k-1} X_{k-1}+W_{k-1} \\
Z_{k}=H_{k} X_{k}+V_{k}
\end{gathered}
$$

$X_{k}$ is the state vector, $\emptyset_{k, k-1}$ is the state transition matrix. $Z_{k}$ is the observation vector, $H_{k}$ is the observation matrix. $W_{k-1}$ and $V_{k}$ denote the system noise and the observation noise. $W_{k-1}$ obeys $N\left(0, Q_{k-1}\right)$ distribution and $V_{k}$ obeys $N\left(0, R_{k}\right)$.

In this paper, $X_{k}=[x, \dot{x}, y, \dot{y}, \theta, \dot{\theta}]^{T}, x$ is the lateral displacement of the current frame relative to the first frame, while $y$ denotes the vertical displacement. $\theta$ denotes the roll angle of the current frame relative to the reference frame. $\emptyset_{k, k-1}$ and $W_{k-1}$ can be denotes as:

$$
\emptyset_{k, k-1}=\left[\begin{array}{cccccc}
1 & 1 & 0 & 0 & 0 & 0 \\
0 & 1 & 0 & 0 & 0 & 0 \\
0 & 0 & 1 & 1 & 0 & 0 \\
0 & 0 & 0 & 1 & 0 & 0 \\
0 & 0 & 0 & 0 & 1 & 1 \\
0 & 0 & 0 & 0 & 0 & 1
\end{array}\right] W_{k-1}=\left[\begin{array}{c}
0 \\
N\left(0, \sigma_{x}\right) \\
0 \\
N\left(0, \sigma_{y}\right) \\
0 \\
N\left(0, \sigma_{\theta}\right)
\end{array}\right]
$$

The $x, y$ and $\theta$ in the state vector are prediction values. In the calculation process, the superscript ${ }^{\wedge}$ is added to distinguish the prediction value from the observation value. The observation equation can be denoted as $[x, y, \theta]^{T}, H_{k}$ and $V_{k}$ can be denoted as:

$$
H_{k}=\left[\begin{array}{llllll}
1 & 0 & 0 & 0 & 0 & 0 \\
0 & 0 & 1 & 0 & 0 & 0 \\
0 & 0 & 0 & 0 & 1 & 0
\end{array}\right] \quad V_{k}=\left[\begin{array}{l}
N\left(0, \sigma_{x}{ }^{o b s}\right) \\
N\left(0, \sigma_{y}{ }^{o b s}\right) \\
N\left(0, \sigma_{\theta}{ }^{o b s}\right)
\end{array}\right]
$$

In $\mathrm{AKF}$, observation noise and prediction noise are considered that the average is not 0 , but is $\hat{q}_{\mathrm{k}}$ and $\hat{r}_{k}$. Then, the state equation (11) and observation equation (12) are given as:

$$
\begin{gathered}
X_{k}=\emptyset_{k, k-1} X_{k-1}+W_{k-1}+\hat{q}_{k-1} \\
Z_{k}=H_{k} X_{k}+V_{k}+\hat{r}_{k}
\end{gathered}
$$

In the equation above, $E\left[\hat{q}_{k-1}\right]=q_{k}, E\left[\hat{r}_{k}\right]=r_{k}$. The other parameters correspond with the equation (11) and (12). Then, the processes of AKF are given as follows:

Step 1: Calculate one-step prediction state $\hat{X}_{k, k-1}$ and noise covariance matrix $P_{k, k-1}$.

$$
\begin{gathered}
\hat{X}_{k, k-1}=\emptyset_{k, k-1} \hat{X}_{k-1}+\hat{q}_{k-1} \\
P_{k, k-1}=\emptyset_{k, k-1} P_{k-1} \emptyset_{k, k-1}{ }^{T}+\hat{Q}_{k-1}
\end{gathered}
$$

Step 2: Update filter gain $K_{k}$ :

$$
K_{k}=P_{k, k-1} H_{k}{ }^{T}\left[H_{k} P_{k, k-1} H_{k}^{T}\right]+\hat{R}_{k-1}
$$

Step 3: Calculate the residual $\varepsilon_{k}$ :

$$
\varepsilon_{k}=Z_{k}-H_{k} \hat{X}_{k, k-1}-\hat{r}_{k-1}
$$

Step 4: Update the state vector and the noise covariance 
matrix:

$$
\begin{gathered}
\hat{X}_{k}=\hat{X}_{k, k-1}+K_{k} \varepsilon_{k} \\
P_{k}=\left(I-K_{k} H_{k}\right) P_{k, k-1}
\end{gathered}
$$

Step 5: Calculate the weigh factor $d_{k}$ :

$$
d_{k}=\frac{1-b}{1-b^{k+1}}
$$

In the above equation, $b$ is the relaxation factor, $b \in$

Step 6: Update $\hat{q}_{k}, \hat{Q}_{k}, \hat{r}_{k}, \hat{R}_{k}$ :

$$
\begin{gathered}
\hat{q}_{k}=\left(1-d_{k}\right) \hat{q}_{k-1}+d_{k}\left(\hat{X}_{k}-\emptyset_{k, k-1} \hat{X}_{k-1}\right) \\
\hat{Q}_{k}=\left(1-d_{k}\right) \hat{Q}_{k-1}+d_{k}\left(K_{k} \varepsilon_{k} \varepsilon_{k}^{T} K_{k}+P_{k}\right. \\
\left.-\emptyset_{k, k-1} P_{k-1} \emptyset_{k, k-1}{ }^{T}\right) \\
\hat{r}_{k}=\left(1-d_{k}\right) \hat{r}_{k-1}+d_{k}\left(Z_{k}-H_{k} \hat{X}_{k, k-1}\right) \\
\hat{R}_{k}=\left(1-d_{k}\right) \hat{R}_{k-1}+d_{k}\left(\varepsilon_{k} \varepsilon_{k}^{T}-H_{k} P_{k, k-1} H_{k}{ }^{T}\right)
\end{gathered}
$$

Consider that the measurement accuracy has a positive correlation with the number of points, $\hat{R}_{k}$ is updated as: $\hat{R}_{k}=\left[\left(1-d_{k}\right) \hat{R}_{k-1}+d_{k}\left(\varepsilon_{k} \varepsilon_{k}{ }^{T}-H_{k} P_{k, k-1} H_{k}{ }^{T}\right)\right] *\left[\frac{E\left(N_{k}\right)}{n_{k}}\right]^{r}$

$r$ is a parameter to indicate the influence of the number of points on the measurement accuracy. The larger $r$ indicates the greater impact of the number of points on measurement accuracy.

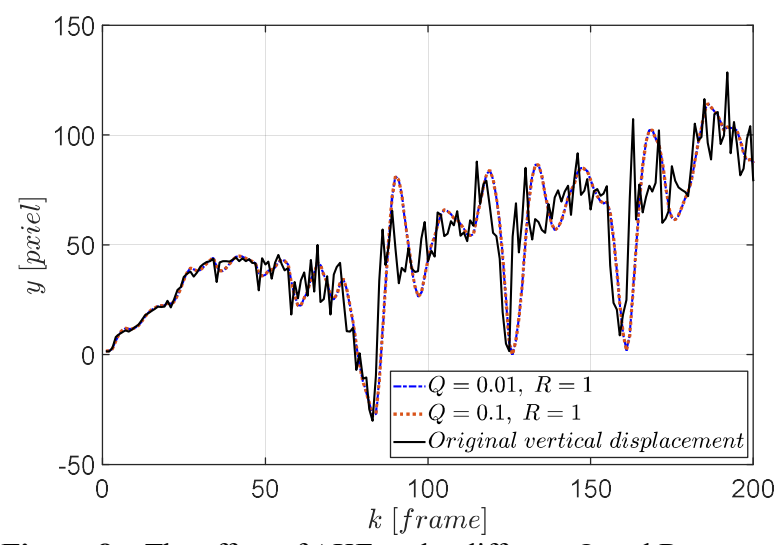

Figure 8 The effect of AKF under different $Q$ and R.

As shown in Figure 8, AKF retains the subjective motion vector and filters out vibration. More importantly, the initial noise setting has almost no effect on the filtering effect, which means that AKF has the ability to adapt to various types of system noise.

\section{Analysis of the effect of electronic image stabilization algorithm}

The effect of EIS is often measured by the peak signal to noise ratio (PSNR), it can be calculated by:

$$
P S N R=10 \times \log _{10}\left(\frac{\left(2^{n}-1\right)^{2}}{M S E}\right)
$$

In the equation above, $M S E$ can be denoted as:

$$
M S E=\frac{1}{M N} \sum_{m=1}^{M} \sum_{n=1}^{N}\left(I_{o}(m, n)-I_{c}(m, n)\right)^{2}
$$

$M$ and $N$ dente the length and width of the frame. $I_{o}(m, n)$ denotes the pixel value of the coordinate point $(\mathrm{m}, \mathrm{n})$ in the original figure, while $I_{c}(m, n)$ denotes that of compensated figure. Figure 9 shows the comparison of PSNR between original video and the compensated video

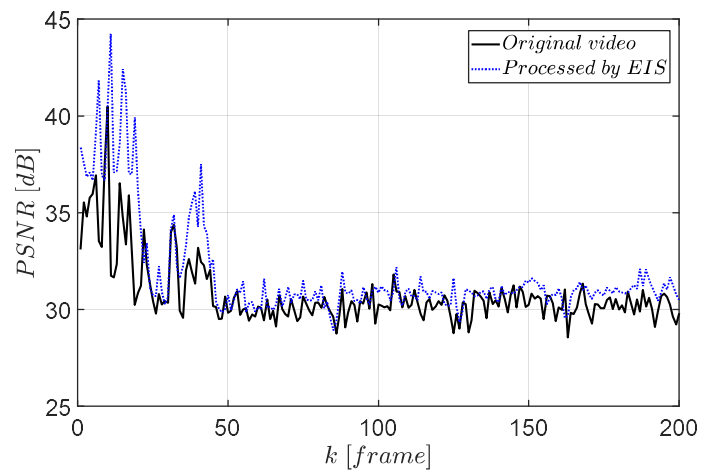

Figure 9 Comparison of PSNR between original video and the compensated video.

The average PSNR is increased by $1.26 \mathrm{~dB}$, which proves the positive effect of EIS. It is worth noting that, $1.2 \mathrm{~dB}$ seems to be less than the results in other papers. Actually, it is meaningless to compare PSNR of different EIS, because if the filter parameters are set to make the filter result smooth enough, PSNR can be greatly improved. However, if doing so, the purpose of preserving the subjective motion vector of the car is lost.

\section{Conclusions}

When the vehicle is running, various working conditions may occur. When concerning some extreme conditions, the image series collected by the camera shake greatly, which affects the subsequent image processing process. To solve this problem, an electronic image stabilization system is developed in this paper for vehicle application. The research conclusions of this paper are listed as follows:

(1) A gasoline model car with remarkable vibration characteristics is built, the harsh road conditions are also established by installing a bump on the wheels. Therefore, such working conditions are more extreme than normal 
scenarios, which makes the electronic image stabilization system developed in this paper more portable.

(2) Considering the possible extreme conditions and real-time processing requirements, the technical route based on feature point matching is selected for this platform. In combination with vehicle demand, ORB algorithm is used in this paper. It is found that the average time of feature point detection is $6.0 \mathrm{~ms}$, the average detected points of each frame is 483.7. The average amount of matched feature point pairs is 223.4. These values all meet the subsequent processing in this paper.

(3) The matching accuracy $A_{m}$ is defined in this paper. Since $A_{m}$ of RANSAC and the improved RANSAC is 0.068 and 0.020 respectively, the improved RANSAC proposed in this paper has better performance. Besides, the processing speed is increased by $32.4 \%$ compared with RANSAC.

(4) The filter based on Sage-Husa adaptive Kalman filter algorithm is adopted in EIS. The update of system noise is improved in this paper considering the amount of detected feature points. The average PSNR is increased by $1.26 \mathrm{~dB}$, which proves the positive effect of EIS.

\section{Declaration}

\section{Acknowledgements}

The authors sincerely thanks to Mr. Tian Li for his critical discussion and reading during manuscript preparation.

\section{Funding}

Supported by National Natural Science Foundation of China (Grant No. 52072072), State Key Laboratory of Mechanical Transmissions (Grant No. SKLMT-KFKT-201713), National Key R\&D Program of China (Grant No. 2016YFD0700905), National Nature Science Foundation (Grant No. 51605087 \& U1664258).

\section{Availability of data and materials}

The datasets supporting the conclusions of this article are included within the article.

\section{Competing interests}

The authors declare no competing financial interests.

\section{Consent for publication}

Not applicable

\section{Ethics approval and consent to participate}

Not applicable

\section{References}

[1] Jean M, James M, Cruickshank, Leandre S. Video-rate image stabilization system $[\mathrm{J}]$. Proceedings of Spie the International Society for Optical Engineering, 1998, 62(6): 313-321.

[2] Mingkhwan E, Khawsuk W. Digital image stabilization technique for fixed camera on small size drone[C]//2017 Third Asian Conference on Defence Technology (ACDT). IEEE, 2017: 12-19.

[3] Rohit Raj, Pooshkar Rajiv, Prabhat Kumar, Manju Khari, Elena Verdú, Rubén González Crespo, Gunasekaran Manogaran, Feature based video stabilization based on boosted HAAR Cascade and representative point matching algorithm[J]. Image and Vision Computing, 2020, 101:103957.

[4] Han C. An Improved Harris Corner Detection Algorithm Based on Adaptive Gray Threshold[C]. Institute of Management Science and Industrial Engineering. Proceedings of 2019 4th International Conference on Automatic Control and Mechatronic Engineering, 2019: 304-308

[5] Lowe D G. Distinctive image features from scale-invariant key points[J]. International Journal of Computer Vision, 2004, 2(60): 91-110.

[6] Bay H, Andreas E, Tinne T, Luc V G. SURF: Speeded Up Robust Features. Computer Vision and Image Understanding[J], 2008, 110(3): 346-359.

[7] Edward R, Tom D. Machine learning for high-speed corner detection[M], European conference on computer vision. Springer, Berlin, Heidelberg, 2006

[8] Kir B, Kurt M, Urhan O. Local Binary Pattern Based Fast Digital Image Stabilization[J]. IEEE Signal Processing Letters, 2014, 22(3): 341-345.

[9] Mikolajczyk K, Schmid C. Indexing based on scale invariant interest points[C]// Proc. of International Conference on Computer Vision. 2001:525-531 vol.1

[10] Ke Y, Sukthankar R. PCA-SIFT: A More Distinctive Representation for Local Image Descriptors[C]// IEEE Computer Society, 2004: 506-513.

[11] Rublee E, Rabaud V, Konolige K, et al. ORB: An efficient alternative to SIFT or SURF[C] //Proceedings of International Conference on Computer Vision. Los Alamitos: IEEE Computer Society Press, 2011, 2564-2571.

[12] Wang Y, Chang R, Chua T W, et al. Video stabilization based on high degree $b$-spline smoothing[C]/Proceedings of the $21 \mathrm{st}$ International Conference on Pattern Recognition (ICPR2012). IEEE, 2012: 3152-3155.

[13] Ren Z, Chen C, Fang M. Electronic image stabilization algorithm based on smoothing 3D rotation matrix[C]// 2017 3rd IEEE International Conference on Computer and Communications (ICCC). IEEE, 2017, pp. 2752-2755.

[14] Cheng X, Hao Q, Xie M. A comprehensive motion estimation technique for the improvement of EIS Methods based on the SURF algorithm and Kalman filter[J]. Sensors, 2016, 16(4): 486.

[15] Lakshya K, Indu S. A Hybrid Filtering Approach of Digital Video Stabilization for UAV Using Kalman and Low Pass Filter[J]. Procedia Computer Science, 2016, 93: 359-366. 
[16] Park R Y, Pak J M , Ahn C K, et al. Image stabilization using FIR filters $[\mathrm{C}] / / 2015$ 15th International Conference on Control, Automation and Systems (ICCAS). IEEE, 2015.

[17] Choi Y W, Kang T H, Lee S G. Development of image stabilization system using extended kalman filter for a mobile $\operatorname{robot}[\mathrm{C}] / /$ International Conference in Swarm Intelligence. Springer, Berlin, Heidelberg, 2010: 675-682.

[18] Yang J, Dan S, Mohamed M. Robust Video Stabilization Based on Particle Filter Tracking of Projected Camera Motion[J]. IEEE Transactions on Circuits \& Systems for Video Technology, 2009, 19(7): 945-954

[19] Zhu J, Li C, Xu J. Digital Image Stabilization for Cameras on Moving Platform[C]// 2015 International Conference on Intelligent Information Hiding and Multimedia Signal Processing. IEEE, 2015, 255-258.

[20] Ioannidis K, Andreadis I. A Digital Image Stabilization Method Based on the Hilbert-Huang Transform[J]. IEEE Transaction on Instrumentation and Measurement, 2012, 61(9): 2446-2457.

[21] Wang J, Zheng S, Du Y, et al. Study on the ORB algorithm in the application of Monocular SLAM[J]. Nanotechnology, 2015, 2(3): 186 .

[22] Montijano E, Martinez S, Sagues C. Distributed Robust Consensus Using RANSAC and Dynamic Opinions[J]. IEEE Transactions on Control Systems Technology, 2014, 23(1):150-163.

[23] Wang Y, Sun Y, Dinavahi V. Robust Forecasting-Aided State Estimation for Power System Against Uncertainties[J]. IEEE Transactions on Power Systems, 2020, 35(1):691-702. 


\section{Figures}

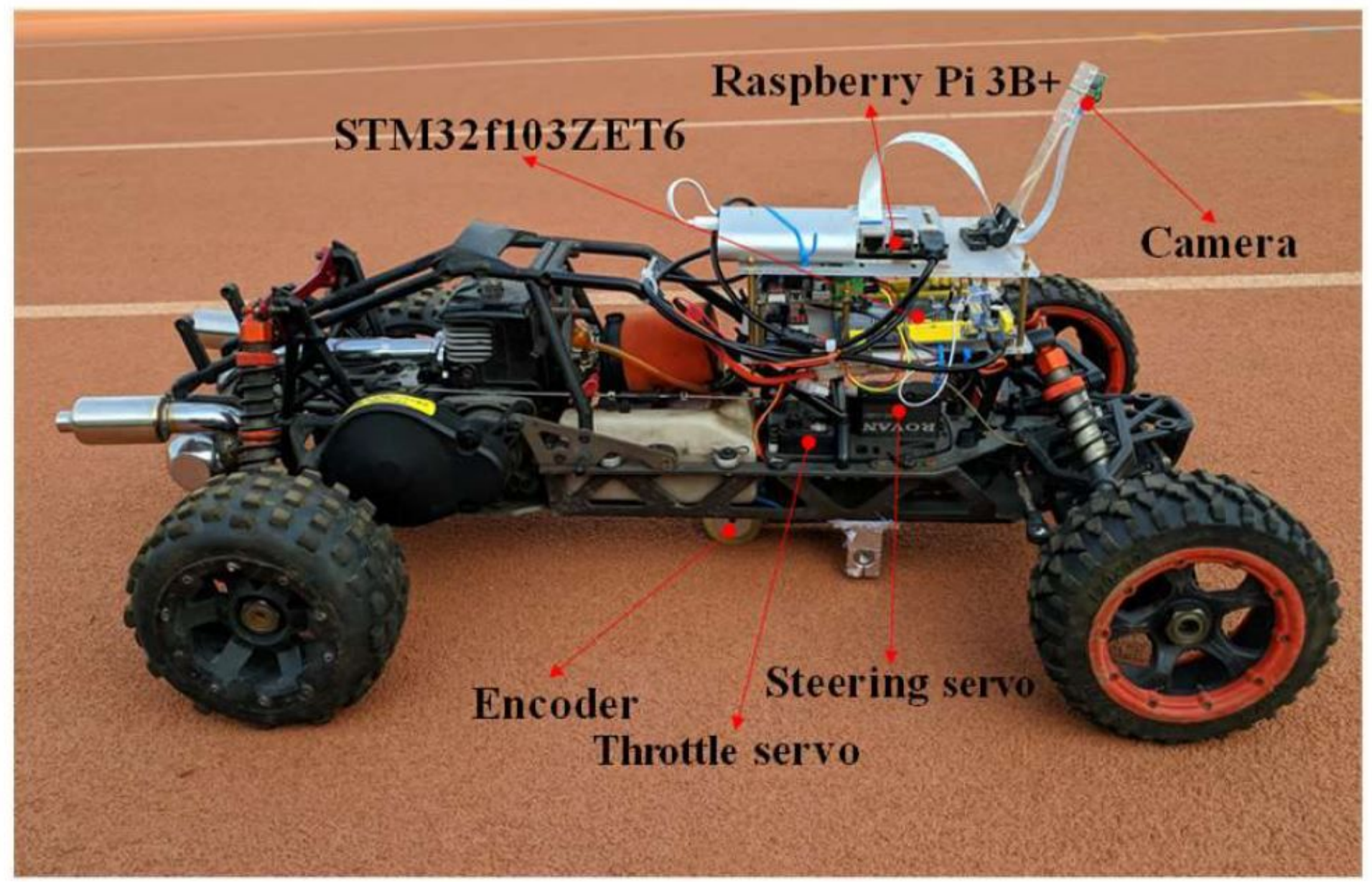

Figure 1

Structural layout of the model car. 


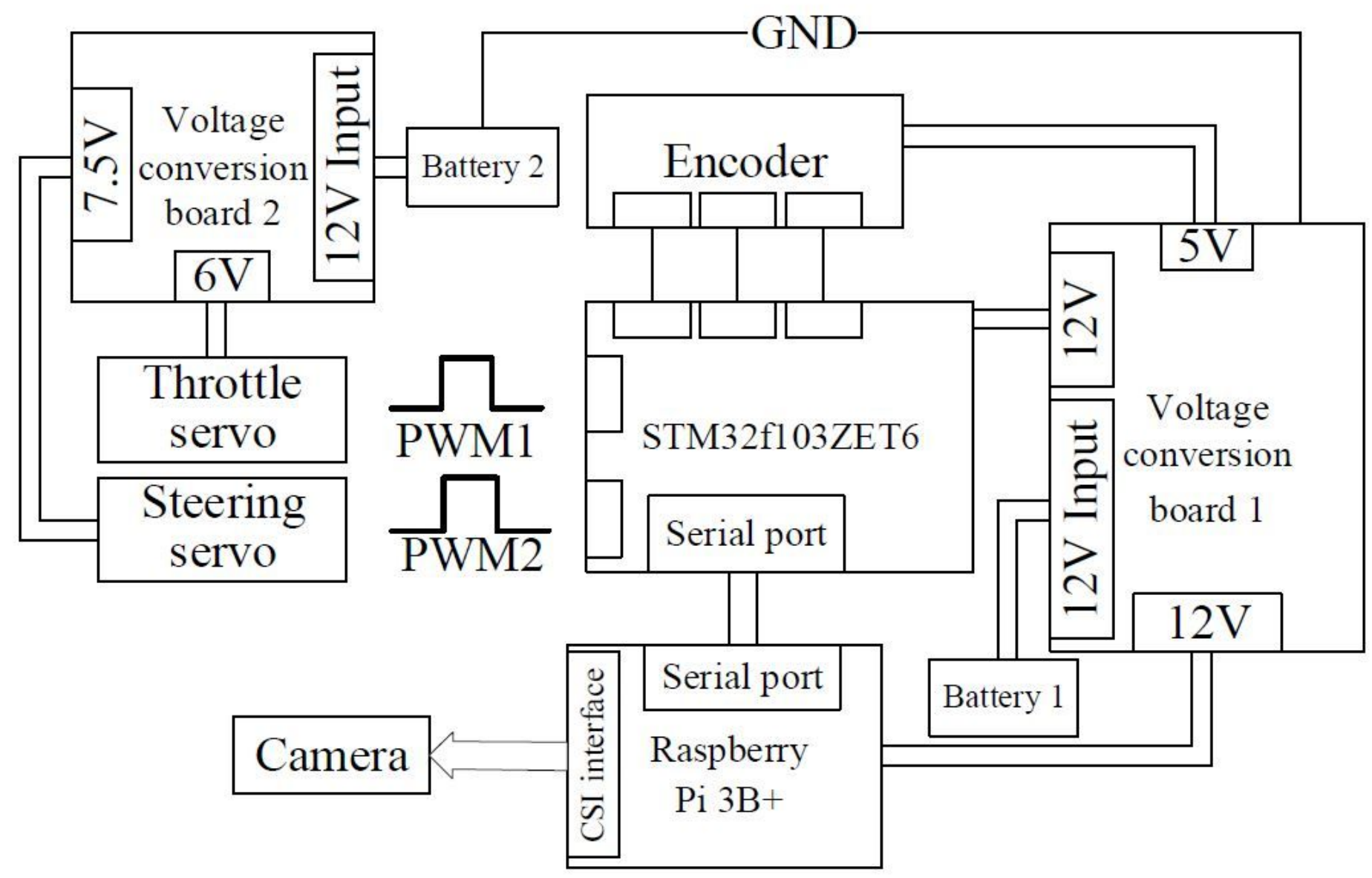

Figure 2

EE architecture of the platform. 

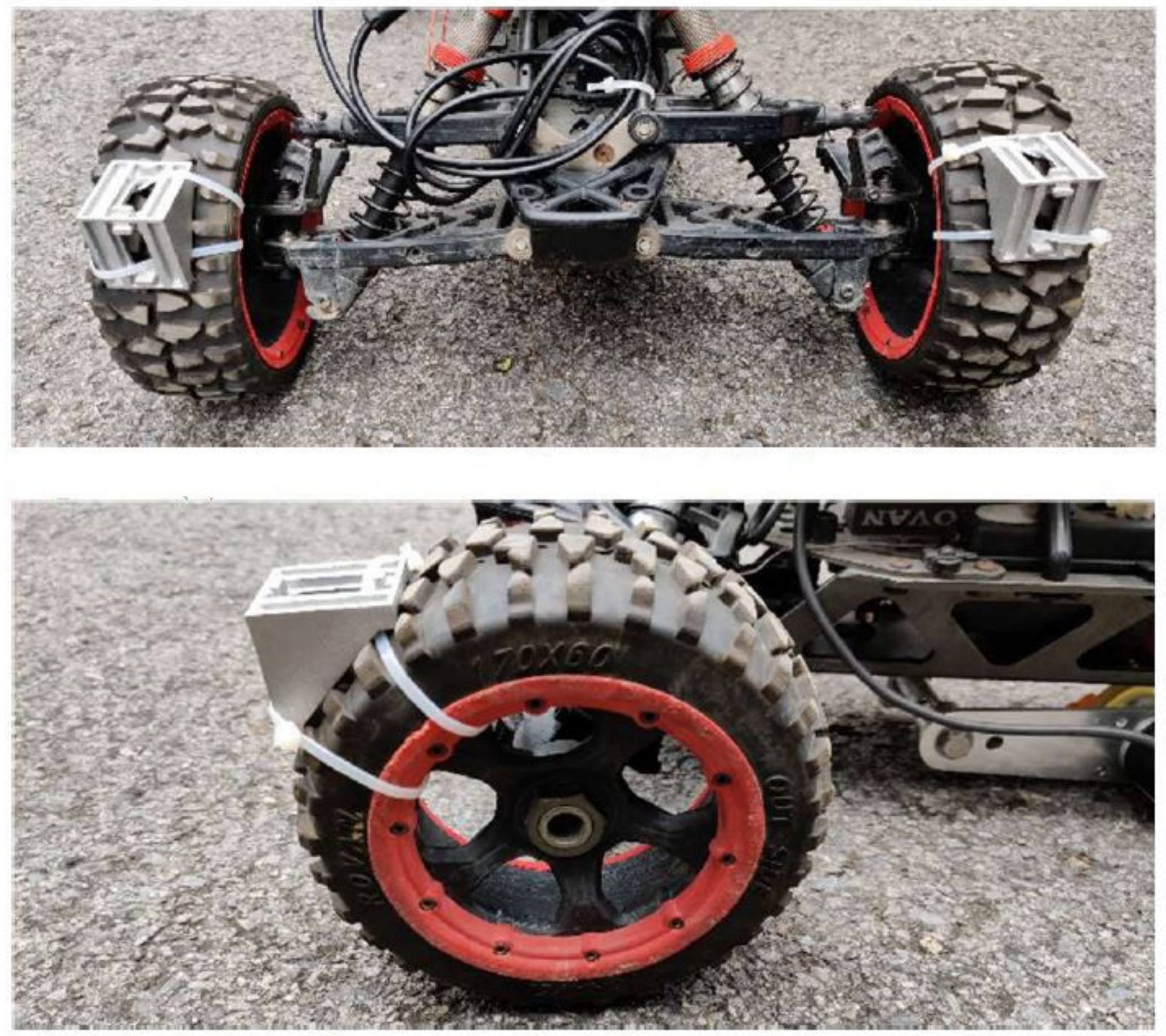

Figure 3

(a) Front wheels of the model car. (b) Left front wheel of the model car. 


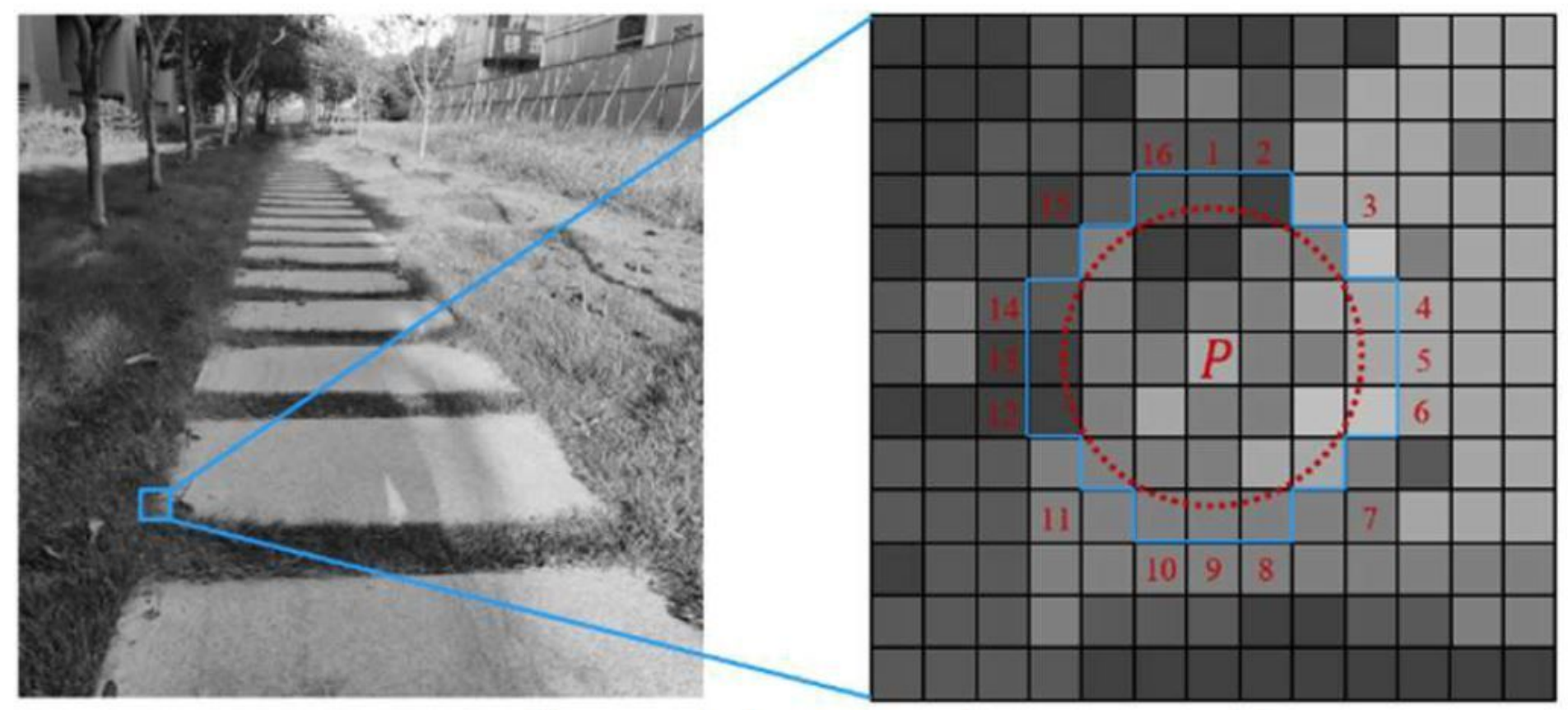

\section{Figure 4}

Corner point and the 16 points around it.

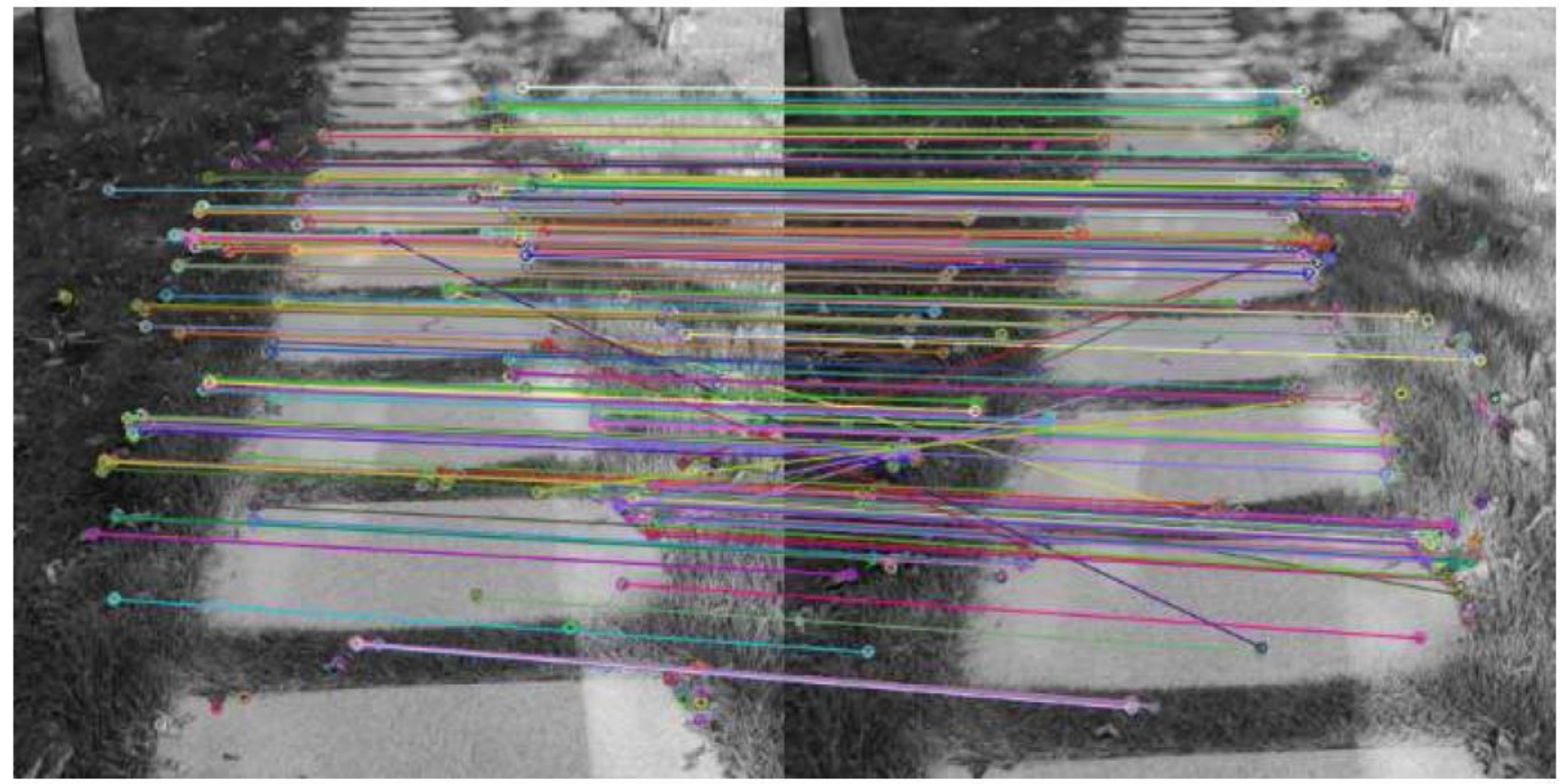

\section{Figure 5}

Feature point detection and matching results. 


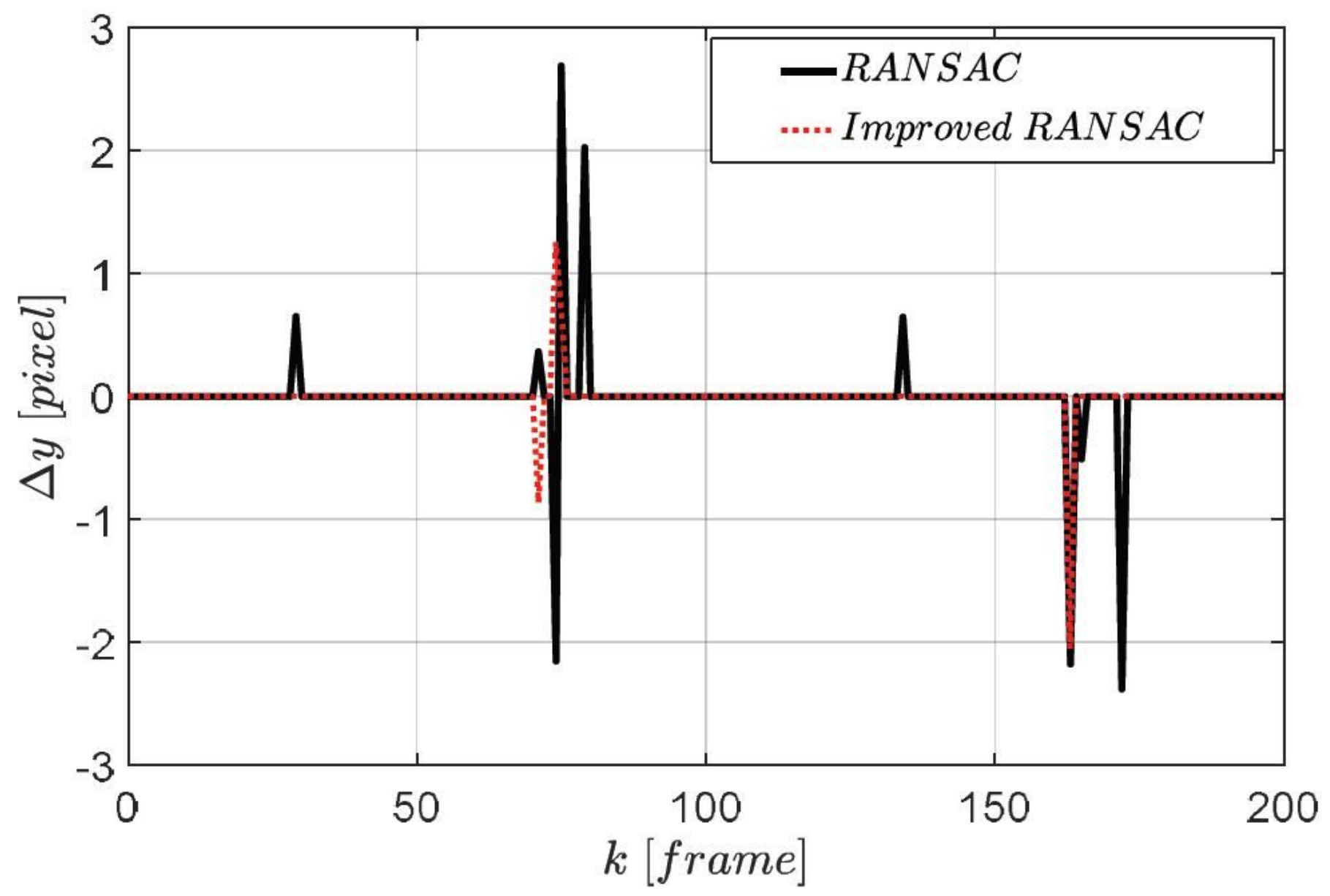

Figure 6

Matching results of the improved RANSAC. 


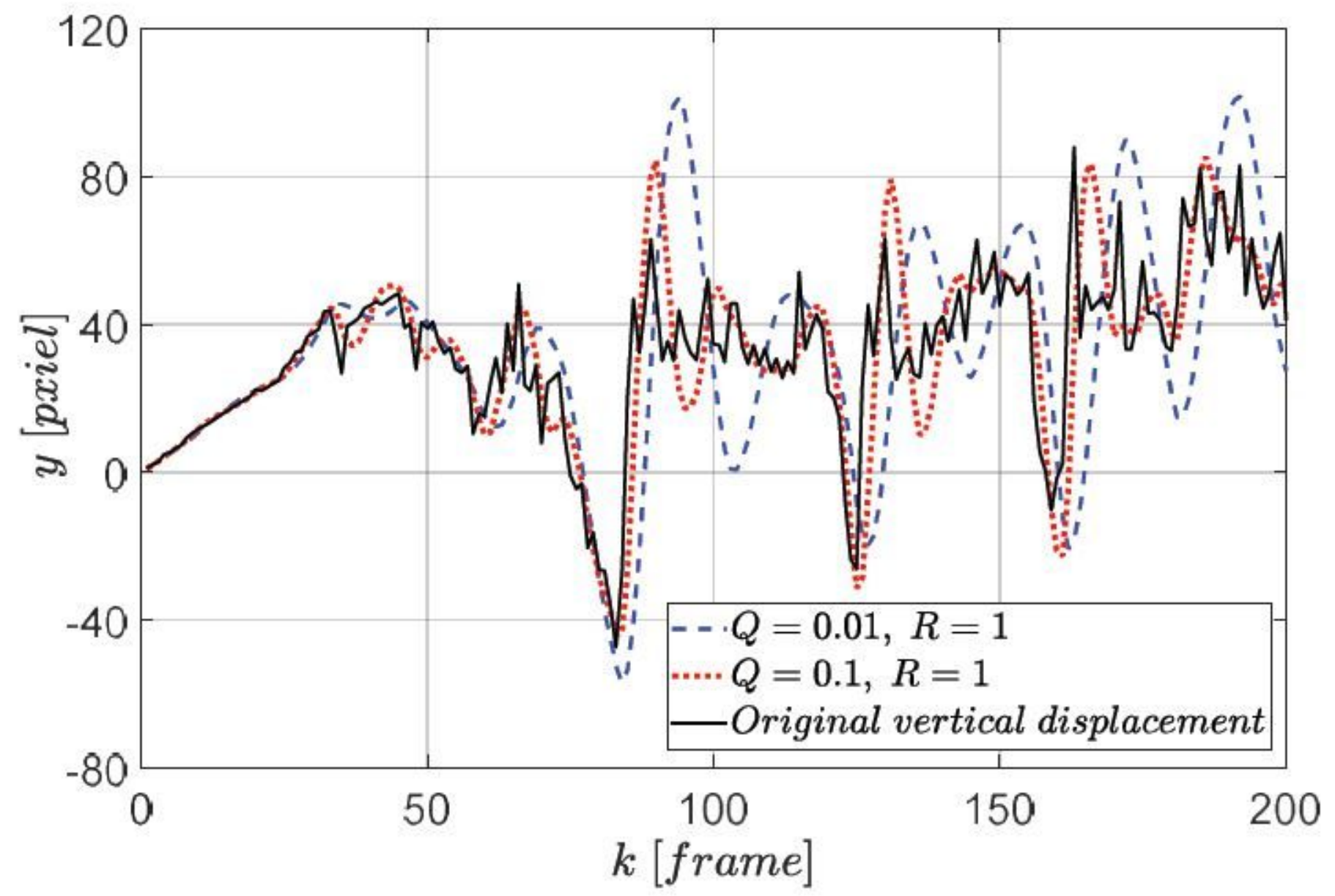

Figure 7

The effect of KF under different $Q$ and $R$. 


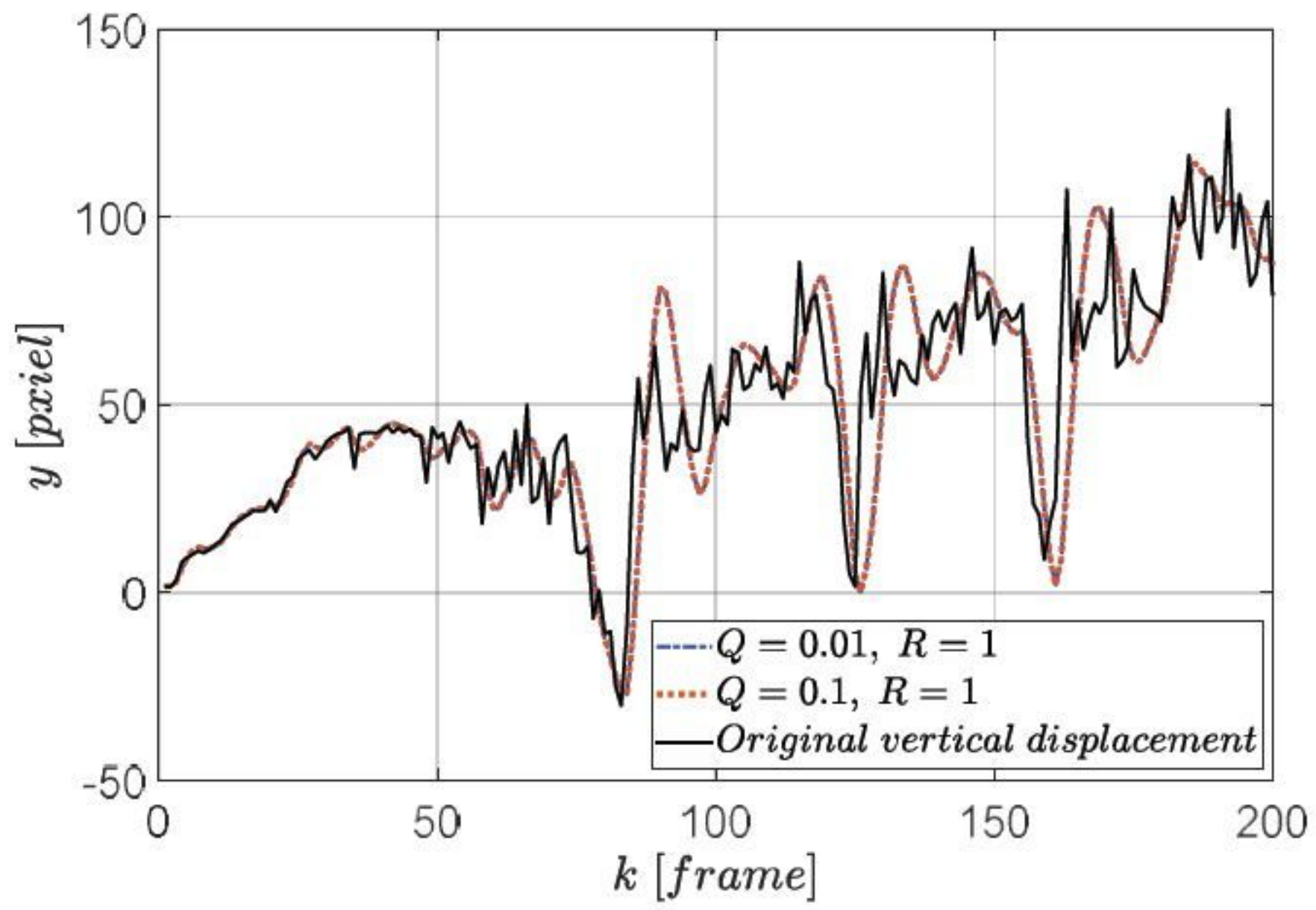

Figure 8

The effect of AKF under different $Q$ and $R$. 


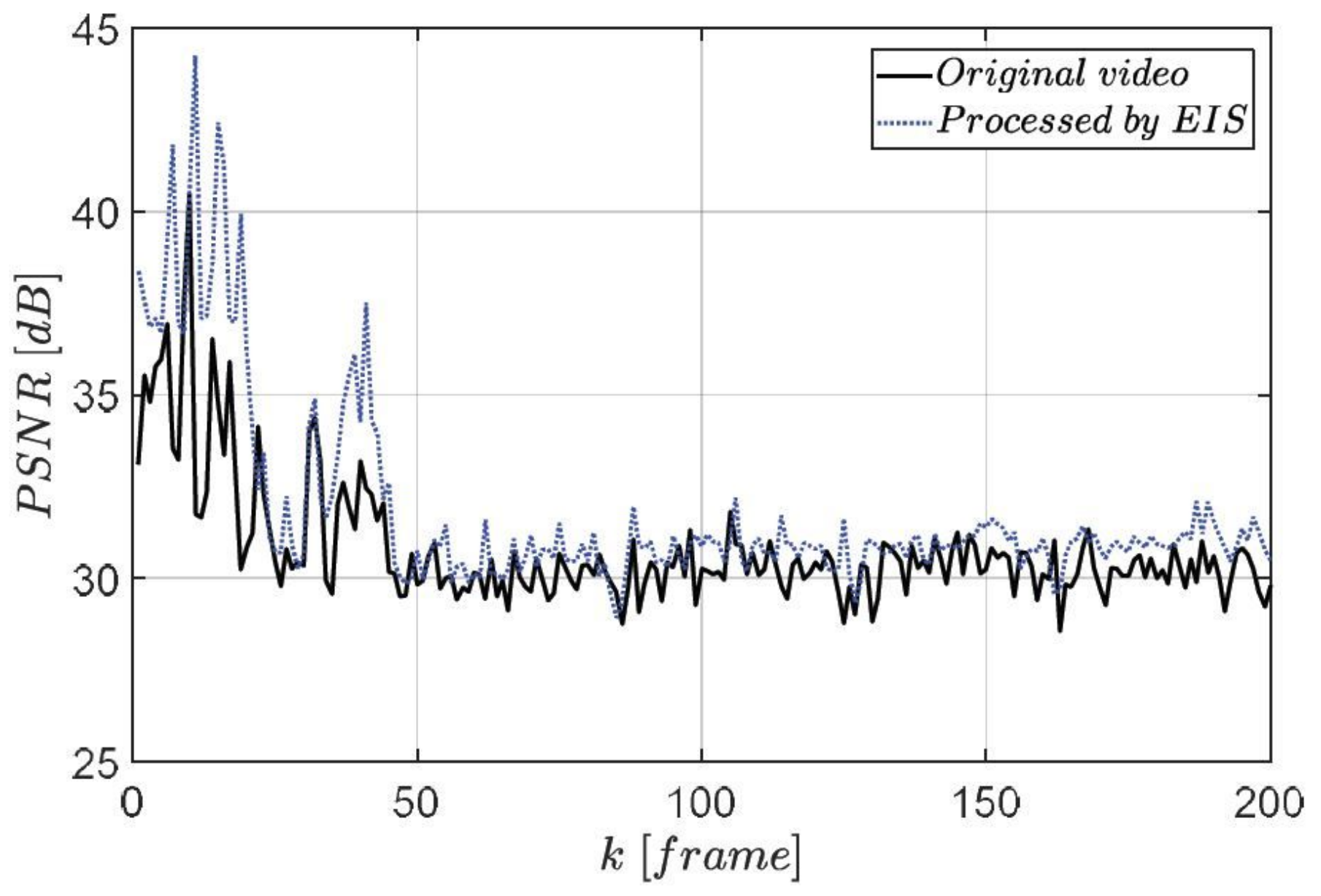

Figure 9

Comparison of PSNR between original video and the compensated video. 